\title{
Empirical Analysis on The Consumption Structure of Urban Residents in Tianjin Based on ELES Model
}

\author{
Ding Xiaoyang \\ Tianjin University of Finance and Economics, Fu Qiang Road, Gangu County, Gansu Province, \\ China \\ gsggdxy@163.com
}

Keywords: ELES Model, Urban Residents, Consumption Structure

\begin{abstract}
Based on the per capital income of urban residents in Tianjin and other data related to the consumption structure, combined with the extended linear expenditure (ELES) model, we can make an empirical analysis of the consumption structure of urban residents in Tianjin in the six years from 2010 2015, and through the calculation of basic consumption expenditure, income elasticity and price elasticity analysis in recent years, we can also learned the dynamic changes of the consumption structure of urban residents in Tianjin. The results show that the consumption demand of Tianjin urban residents is not strong enough, the consumption structure is not reasonable, the proportion of consumption expenditure is low, and household equipment and transportation are more sensitive to income and their own price.
\end{abstract}

\section{Introduction}

Consumption is the final part of the socialist expansion of reproduction, the consumption process through the reverse transmission, which greatly affects the production structure and industrial structure, their adjustment to the consumption structure as a prerequisite. It is helpful for the government to formulate reasonable policies to optimize the consumption structure, to adjust the industrial development, to improve the people's living standard and to promote the healthy operation of the economy by studying and analyzing the residents' consumption structure and its changing trend. In the consumption structure analysis, we usually use the Linear Expenditure System (LES) model or the Extended Linear Expenditure System (ELES) model to analyze the consumer demand structure and the changing trend of the household for all kinds of consumer goods. Among them, ELES model is currently in the consumption structure analysis and prediction of the more advanced economic measurement method.

\section{The Construction of ELES Model}

ELES model is a kind of demand function which is obtained by adding the saving factor to the linear expenditure system model (LES model), which is the deformation of linear expenditure system model (LES model). The model concludes that the demand for a particular commodity is determined by the amount of income and the price of a given commodity in a given period. And the assumption that the demand for goods by the basic needs and more than basic needs beyond the additional needs of two parts, of which the basic needs of people living is necessary, it has nothing 
to do with the amount of income. Even without income people will find ways to meet this part of the demand. So by the basic needs and the proportion of additional demand, we can also get the level of consumption and consumption structure of the information.

Next,we give the ELES model :

$$
V_{i}=P_{i} X_{i}+\beta_{i}\left[Y-\Sigma P_{i} X_{i}\right]
$$

$V_{i}$--Consumption expenditure on the i commodity.

$X_{i}$--The basic demand for the i commodity.

$\beta_{i}$--The marginal propensity to consume for the i commodity.

$Y$--The total income of the family.

We deform the above equation, then can get:

$$
V_{i}=\left[P_{i} X_{i}-\beta_{i} \Sigma P_{i} X_{i}\right]+\beta_{i} Y
$$

Let $a_{i}=P_{i} X_{i}-\beta_{i} \Sigma P_{i} X$, then we can get:

$$
V_{i}=a_{i}+\beta_{i} Y
$$

The equation (3) is a unitary linear equation,As long as we know the data of $V_{i}$ and $Y$, we can estimate the values of $a_{i}$ and $\beta_{i}$ according to the least square method.

Let the two sides of the equation $a_{i}=P_{i} X_{i}-\beta_{i} \Sigma P_{i} X$ are summed according to i,we can get $\Sigma P_{i} X_{i}=\frac{\Sigma a_{i}}{1-\Sigma \beta_{i}}$,replace it in $a_{i}=P_{i} X_{i}-\beta_{i} \Sigma P_{i} X$, we can get

$$
P_{i} X_{i}=a_{i}+\frac{\beta_{i} \Sigma a_{i}}{\left(1-\beta_{i}\right)}
$$

It is also possible to compute the income elasticity and the price elasticity of demand or consumption expenditure:

$$
\eta_{i}=b_{i} \frac{Y}{V_{I}}
$$

$$
\eta_{i j}=\left(1-b_{i}\right) \frac{P_{i} X_{i}}{V_{I}}-1
$$

The consumption structure is the proportion of different goods or services in consumption, which includes food, clothing, household equipment and services(equ), health care, transportation(tra), communications and entertainment(ent), houses, others. According to the Tianjin Bureau of Statistics on urban household sample survey standards, in the study of consumer spending, the general urban residents is divided into five income classes, They are: the low income households, the below average households, the medium income households, the above average income households and the high income households. Therefore, the author uses the cross-section data of consumption structure of urban residents in Tianjin from 2010 to 2015, and can calculate the basic consumption expenditure, income elasticity and self-price elasticity by least-squares estimation of equation parameters.

\section{Tianjin Urban Residents Consumption Structure of The Empirical analysis}

\subsection{The Marginal Propensity to Consume Analysis.}

The marginal propensity to consume refers to the proportion of residents spending on living expenses for each additional unit of income after satisfying the basic consumption demand.The 
parameters of the formula are estimated by least squares method using the $5 \times 7$ section data of Tianjin .We can obtain the marginal propensity to consume and the total marginal propensity to consume of all types of commodities in each year.(see Table 1).

Table1 Estimation of $\beta$ i in ELES Model of Tianjin Urban Residents from 2010-2015

\begin{tabular}{|c|c|c|c|c|c|c|c|c|c|}
\hline year & food & cloth & equ & health & tra & ent & house & others & total \\
\hline 2010 & 0.103 & 0.048 & 0.071 & 0.051 & 0.067 & 0.055 & 0.072 & 0.028 & 0.495 \\
\hline 2011 & 0.112 & 0.053 & 0.065 & 0.049 & 0.074 & 0.064 & 0.081 & 0.035 & 0.533 \\
\hline 2012 & 0.108 & 0.056 & 0.077 & 0.050 & 0.069 & 0.067 & 0.075 & 0.033 & 0.535 \\
\hline 2013 & 0.105 & 0.051 & 0.068 & 0.051 & 0.072 & 0.058 & 0.081 & 0.031 & 0.517 \\
\hline 2014 & 0.101 & 0.053 & 0.073 & 0.057 & 0.081 & 0.062 & 0.112 & 0.029 & 0.568 \\
\hline 2015 & 0.98 & 0.052 & 0.072 & 0.055 & 0.075 & 0.068 & 0.108 & 0.033 & 0.567 \\
\hline
\end{tabular}

From Table 1, in general, from 2010 to 2015, the total marginal propensity to consume of urban residents has been increasing slowly, reaching a peak of 0.568 in 2014, that is, every additional 100 yuan of income, 56.8 yuan for consumption, 43.2 yuan To increase savings. This shows that people's consumption concept is gradually changing, there will be more income for consumption rather than savings.But the income of urban residents in Tianjin for the proportion of consumption is still relatively low, consumer demand has not been strong enough, the role of consumption to stimulate the economy is too slow for Tianjin .Therefore, how to encourage people to increase consumption and change the traditional concept of ignoring consumption is very important .

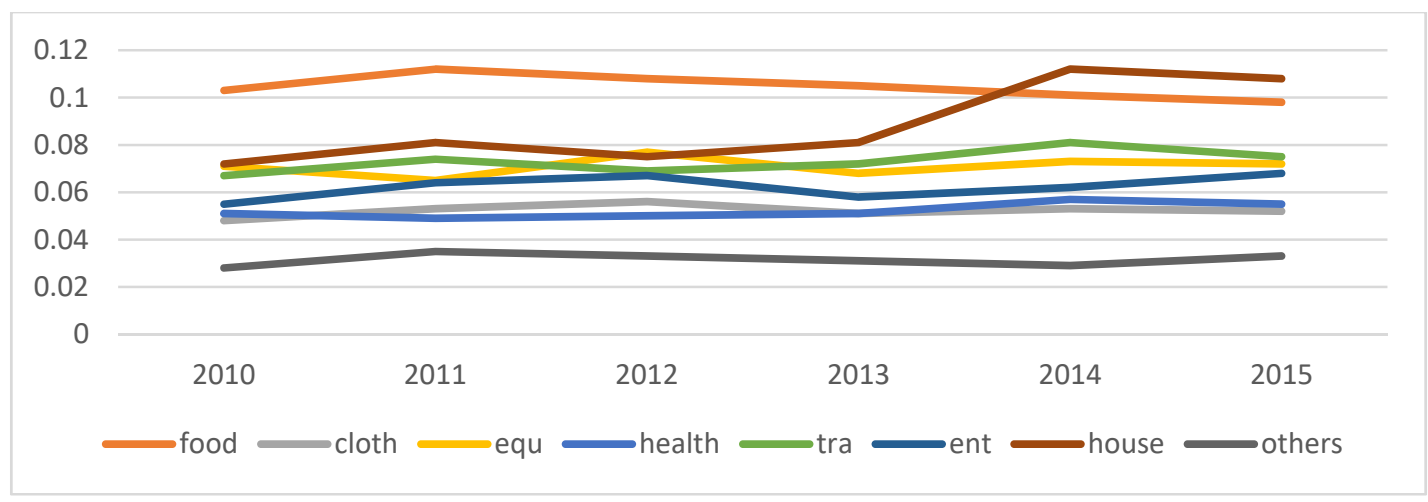

Fig 1 Estimation of $\beta$ i in ELES Model of Tianjin Urban Residents From 2010-2015

From the Fig 1 and Table 1, in 2010 2015 years, the top three of the marginal propensity to consume is food, houses, and household equipment. this shows that in recent years Tianjin new urban residents income is mainly used for food, housing and household equipment spending(of course, part of the added value to take into account the impact of rising prices); In 2014, houses in the marginal propensity to consume suddenly has been greatly improved, which is likely due to the rapid rise in housing prices.we can see the food and the marginal propensity to consume gradually began to decline and the transportation and communication, culture and entertainment gradually increase the marginal propensity to consume .This shows that, after meet the food, clothing, living needs, urban residents in Tianjin has gradually shifted to consumption to improve the quality of life of transportation and communication, education and entertainment services spending, consumption structure gradually become more reasonable. 


\subsection{Analysis of Basic Consumption}

The basic definition of the basic consumer demand expenditure is defined as the ability to pay for the basic consumption of goods and services in order to ensure the normal reproduction of the labor force.Take the estimated value from the least squares method into the equation, calculated the basic consumption of various types of goods consumption level of expenditure and the level of total annual expenditure of basic consumption from 2010 to 2015 (see table 2.)

The basic needs of consumers in order to meet the basic needs of the consumer demand, the basic needs of the total expenditure accounted for an average of actual consumption can reflect the degree of contrast residents living consumption and enjoyment of consumption.From 2010 to 2015, the ratio continued to decline, (see table 2 ) respectively: $55.7 \%, 52.3 \%, 50.5 \%, 49.2 \%, 47.9 \%$ 、 $48.3 \%$.After 2012 the proportion dropped to below 50\%. This shows that the living standards of urban residents in Tianjin have been significantly improved in recent 3 years.

Table 2 Basic Consumption Expenditure of Urban Residents in Tianjin From 2010-2015 (unit:yuan)

\begin{tabular}{|c|c|c|c|c|c|c|c|c|c|c|}
\hline year & food & cloth & slp & med & tra & ent & houses & 0thers & $\begin{array}{c}\text { Total } \\
\text { (basic) }\end{array}$ & proportion \\
\hline 2010 & 3308.8 & 873.2 & 623.8 & 710.5 & 1367.1 & 1058.0 & 899.9 & 383.6 & 9224.9 & 0.557 \\
\hline 2011 & 3484.9 & 917.9 & 614.3 & 740.3 & 1411.8 & 1106.7 & 922.3 & 437.6 & 9635.8 & 0.523 \\
\hline 2012 & 3708.5 & 950.1 & 581.4 & 786.0 & 1557.1 & 1138.4 & 936.4 & 454.4 & 10112.3 & 0.505 \\
\hline 2013 & 3908.0 & 959.7 & 593.2 & 833.6 & 1706.7 & 1157.9 & 1027.6 & 495.6 & 10682.3 & 0.492 \\
\hline 2014 & 3865.3 & 982.3 & 664.4 & 824.5 & 1491.6 & 964.2 & 2515.4 & 327.0 & 11634.8 & 0.479 \\
\hline 2015 & 4080.2 & 1035.7 & 769.4 & 912.0 & 1643.7 & 1102.5 & 2737.3 & 388.0 & 12668.9 & 0.483 \\
\hline
\end{tabular}

By the data in Table 2, we can get a monthly average of Tianjin urban residents' basic consumption expenditure, and according to the monthly basic consumption expenditure will probably determine the level of basic social relief .From the data in Table 2, we can get the average of basic monthly total expenditures of Tianjin urban residents were 768.7(9224.9/12, and so on), 803.0, 842.7, 890.2, 969.6and 1055.74(yuan) from 2010-2015. When the income level of residents is lower than the level of the total expenditure of basic consumption, it means that the residents can not guarantee the basic living needs, so the Tianjin municipal government needs to subsidize these people in order to maintain their normal life. We can see that the analysis of the basic consumer demand is of great significance in social security and welfare research . The government should ensure that the minimum income level of each person is above the basic consumer demand.

\subsection{An Analysis of Income Elasticity and Price Elasticity of Demand}

The elasticity of income represents the degree of response to the change in the amount of consumer demand in a given period of time because the change of income.Or that the percentage change in commodity demand in a certain period of time when the 1/100 changes in income caused by the consumer.The price elasticity of demand is a measure of the extent to which a change in the quantity demanded of a commodity affects the price of a commodity over a given period of time.

In general, the income elasticity of the various types of commodities in Table 4 shows that the income elasticity of food is the lowest in all years, which has remained at 0.35 . The income sensitivity is not strong, even if the income growth, food consumption growth will not be great, showing the characteristics of rigid consumption of food consumption; Among the other consumption items, the income elasticity of clothing and housing remained basically fluctuated around 0.8, while the income elasticity of health care, culture and entertainment was generally around 0.6, and the impact of income fluctuation on them was poor. Specific in the year 2015, income for every $1 \%$ increase ,household equipment spending will increase $11.13 \%$, traffic and 
communication will increase $11.89 \%$, an increase of more than revenue growth.

Compared to the demand elasticity of income, demand for the price elasticity are negative, indicating that the price of various types of consumer goods have a negative effect on their demand. These two types of consumption expenditure are more sensitive to price changes and have stronger price effect, and the absolute maximum of price elasticity is 0.672 and 0.655 respectively in 2015.The price elasticity of food and entertainment is mostly above - 0.3 , showing price rigidity, which is only 0.366 and -0.415 in 2014 , respectively.This is basically consistent with the demand elasticity analysis of income. With the rapid economic growth in Tianjin and the rising incomes of urban residents in Tianjin, the desire to increase household appliances and transportation and communications will be even stronger, and they will be greatly affected by their own prices.

Table 4 Income Elasticity and Price Elasticity of Urban Residents' Consumption in Tianjin From 2010-2015

\begin{tabular}{|c|c|c|c|c|c|c|c|c|c|}
\hline year & genre & food & cloth & equ & health & tra & ent & house & others \\
\hline \multirow{2}{*}{2010} & $\eta_{i}$ & 0.355 & 0.896 & 1.362 & 0.762 & 1.080 & 0.438 & 0.872 & 1.047 \\
\cline { 2 - 10 } & $\eta_{i j}$ & -0.279 & -0.508 & -0.701 & -0.433 & -0.599 & -0.266 & -0.519 & -0.551 \\
\hline \multirow{2}{*}{2011} & $\eta_{i}$ & 0.372 & 0.854 & 1.082 & 0.724 & 1.130 & 0.571 & 0.791 & 1.157 \\
\cline { 2 - 10 } & $\eta_{i j}$ & -0.286 & -0.453 & -0.542 & -0.416 & -0.574 & -0.320 & -0.439 & -0.579 \\
\hline \multirow{2}{*}{2012} & $\eta_{i}$ & 0.367 & 1.028 & 1.199 & 0.807 & 1.048 & 0.568 & 0.755 & 1.224 \\
\cline { 2 - 10 } & $\eta_{i j}$ & -0.286 & -0.427 & -0.606 & -0.464 & -0.560 & -0.305 & -0.443 & -0.616 \\
\hline \multirow{2}{*}{2013} & $\eta_{i}$ & 0.317 & 0.814 & 1.138 & 0.520 & 1.214 & 0.492 & 0.998 & 1.075 \\
\cline { 2 - 10 } & $\eta_{i j}$ & -0.238 & -0.414 & -0.540 & -0.315 & -0.633 & -0.251 & -0.533 & -0.519 \\
\hline \multirow{2}{*}{2014} & $\eta_{i}$ & 0.458 & 0.908 & 1.216 & 0.585 & 1.059 & 0.649 & 0.773 & 1.407 \\
\cline { 2 - 9 } & $\eta_{i j}$ & -0.366 & -0.572 & -0.750 & -0.421 & -0.646 & -0.415 & -0.502 & -0.829 \\
\hline \multirow{2}{*}{2015} & $\eta_{i}$ & 0.383 & 0.873 & 1.113 & 0.591 & 1.189 & 0.617 & 0.837 & 1.388 \\
\cline { 2 - 9 } & $\eta_{i j}$ & -0.298 & -0.435 & -0.672 & -0.432 & -0.655 & -0.375 & -0.497 & -0.821 \\
\hline
\end{tabular}

\section{Conclusion and Policy Suggestion}

According to the data analysis of 2010-2015, this paper draws the following conclusions:

Tianjin as the economic center in the north, with the development of Binhai New Area to drive rapid economic growth in the surrounding areas, the goal is to build "the third pole."in China's economic growth, "the third pole."As a result, the state will give Tianjin a variety of preferential and preferential policies, such as the national counter trading market settled in Tianjin Binhai New Area. With the national policy guidance, combined with the efforts of all sectors of Tianjin, Tianjin, the future economic growth will be high.Based on this macro background, combined with the conclusions, I propose the following policy recommendations:

Accelerate the construction of social security system, improve the consumer confidence, promote consumption. To improve the social security system and enhance people's confidence in the future livelihood guarantee, the saving part of the basic needs such as unemployment and medical care will be reduced, and the residents will naturally increase their consumption expenditure.

Focusing on the release of middle and high income groups spending power. The spending power of middle and high income groups is very strong. The government should develop multi-channel consumption through policy guidance and encourage them to expand consumption. 
Seize the opportunity of economic development, improve the income level of residents, in particular, increase the income of low-income groups. Government relief of low - income families is only short - term behavior, the Government should seize the opportunity of Tianjin great development investment for the community to create more employment opportunities and guide low - income groups employment.

Actively cultivate new consumption hot spots, making it a new round of economic growth point.Elastic analysis shows that in the future people's desire to increase the use of household appliances and transportation is more intense.The government should grasp the new consumption hot spots in time, provide a good macro policy environment and promote its development.

\section{References}

[1] Stone R.Linear expenditure systems and demand analysis[J]. Economic Journal,1954, 64:511-527

[2] Lluch C.The extended linear expenditure system[J]. European Economic Review,1973,14:(1)21-32

[3] National Bureau of Statistics. China Statistical Yearbook. Beijing: China Statistics Press, 2010-2016.

[4] Zou Zheng-long.Effects of Income Growth and Consumption Structure [J]. Business Research, 2015 (3): $23-25$

[5] Xu Hong-shui.Comparative Analysis of Consumption Structure of Chinese Urban and Rural Residents Based on ELES. Shanghai Finance, 2014 (10)

[6] Sun He, Ren Jin-zheng.Empirical Analysis of Food Consumption Structure of Chinese Urban Residents Based on ELES Model [J].Industry Outlook, 2014 (7): 70-74. 